\title{
MEJORAMIENTO DEL CONTROL GLUCÉMICO DE PACIENTES DIABÉTICOS DEL CANTÓN RIOBAMBA.
}

\author{
IMPROVEMENT OF THE GLUCEMIC CONTROL OF DIABETIC PATIENTS OF THE \\ CANTÓN RIOBAMBA.
}

\author{
Raquel Robalino*(1), Gladys Cabay $^{(2)}$, Miriam Piray(1), María Robalino ${ }^{(1)}$, Patricio \\ Chicaiza $^{(3)}$. \\ (I) Hospital Provincial General Docente Riobamba, Riobamba-Ecuador. \\ (2) Instituto Superior Tecnológico de Riobamba, Riobamba - Ecuador. \\ (3) Unidad Educativa Capitán Edmundo Chiriboga, Riobamba - Ecuador.
}

Email:umsmwork74@gmail.com

https://doi.org/10.33789/talentos.7.2.130

\begin{abstract}
Resumen. La diabetes mellitus constituye una de las enfermedades crónicas no transmisibles que mayor morbimortalidad representa a nivel mundial. Las complicaciones de la diabetes mellitus se originan secundariamente al mantenimiento de niveles elevados de glucemia en sangre. La finalidad de esta investigación es implementar una intervención terapéutica para elevar el control glucémico de los pacientes con diabetes mellitus. Para ello se implementó una intervención terapéutica que incluyó elementos educativos, nutricionales y de adecuación farmacológica en un universo de 81 con historia previa de 2 controles glucémicos admisibles o inadecuados; la muestra quedó conformada por 73 pacientes. Se determinó el estado nutricional de los pacientes, el nivel de conocimiento sobre autocuidado; se utilizó la prueba de Rangos con Signos de Wilcoxon para identificar cambios en el control glucémico a partir de la intervención aplicada. Los resultaros mostraron que el 80,82\% de los casos tenía diagnóstico de diabetes mellitus tipo 2, con un tiempo de evolución de la enfermedad predominante entre 5 y 10 años $(65,75 \%)$ y el 83,56\% de los pacientes refirieron al menos una complicación de la enfermedad. En consecuencia, la intervención terapéutica aplicada provocó cambios estadísticamente significativos en el control glucémico de los pacientes con diagnóstico de diabetes mellitus. También se elevó el nivel de conocimiento sobre autocuidado, la adherencia terapéutica y se mejoró el estado nutricional de los pacientes investigados.
\end{abstract}

Recibido: 13 de junio de 2020

Aceptado: 26 de agosto de 2020

Publicado como artículo científico en la Revista de Investigación Talentos VII (2), 1-10 
Palabras clave. Control glucémico; Diabetes Mellitus; Diabetes mellitus tipo 2; Intervención educativa; Trastorno nutricional.

Abstract. Diabetes mellitus is one of the chronic non-communicable diseases that represents the highest morbidity and mortality worldwide. The complications of diabetes mellitus originate secondary to the maintenance of high blood glucose levels. The purpose of this research is to implement a therapeutic intervention to increase glycemic control in patients with diabetes mellitus. For this, a therapeutic intervention was implemented that included educational, nutritional and pharmacological adequacy elements in a universe of 81 with a previous history of 2 admissible or inadequate glycemic controls; the sample was made up of 73 patients. The nutritional status of the patients, the level of knowledge about self-care; The Wilcoxon Sign Ranges test was used to identify changes in glycemic control from the intervention applied. The results showed that $80.82 \%$ of the cases had a diagnosis of type 2 diabetes mellitus, with a predominant time of evolution of the disease between 5 and 10 years $(65.75 \%)$ and $83.56 \%$ of the patients referred at least one complication of the disease. Consequently, the applied therapeutic intervention caused statistically significant changes in glycemic control in patients with a diagnosis of diabetes mellitus. The level of knowledge about self-care, therapeutic adherence, and the nutritional status of the investigated patients improved as well.

Keywords: Glycemic control; Mellitus diabetes; Diabetes mellitus type 2; Intervention; Nutritional disorder.

\section{INTRODUCCIÓN}

La diabetes mellitus (DM) es considerada como una enfermedad crónica no transmisible que genera un variado número de complicaciones con una elevada morbimortalidad mundial (Bell Castillo, 2017). La DM ha sido considerada por la Organización Mundial de la Salud como la epidemia del XXI, pasando de un total de 30 millones de personas diagnosticadas con la enfermedad en el año 1995 a un total de 347 millones en el año 2018 y se estima que las cifras ronden los 366 millones para el año 2020 (Castañeda Bajaña, et al, 2019).

En Ecuador, según cifras ofrecidas por el Instituto Nacional de Estadística y Censo (INEC) la prevalencia de la DM en el año
2018 se estimó en un 10,7\%, constituyendo el $28 \%$ y con una tasa de mortalidad de 30 pacientes por cada 100.000 habitantes (INEC, 2018). Durante el año 2016 la DM constituyó la tercera causa de mortalidad en pacientes masculinos y la segunda en féminas representando el $6,17 \%$ y $8,19 \%$ respectivamente del total de defunciones por sexo (INEC, 2016).

En el Hospital Provincial General Docente de Riobamba (HPGDR), según su departamento de estadística, la DM constituyó en el año 2017 la principal causa de morbimortalidad, presentándose 223 pacientes que representó el $13 \%$ de los ingresos hospitalarios (HPGDR, 2018). Lo que permite afirmar que la DM, como enfermedad sistémica, constituye uno de los problemas de salud priorizados del 
contexto de la investigación.

Estudios internacionales coinciden en señalar el aumento mantenido de los valores de glucemia como el mecanismo etiopatogénicoy fisiopatológico de la aparición y perpetuación de las complicaciones de la DM (Alpízar, et al, 2017). Estas generan daño, disfunción y falla de varios órganos y sistemas, como es el caso de riñones, corazón, pulmones, piel, vasos sanguíneos, sistema nervioso central y periférico y varios órganos de los sentidos; que generan distintos grados de discapacidad y disminución de la percepción de calidad de vida relacionada con la salud (Martín Alfonso, 2018). La Asociación Latinoamericana de Diabetes (ALAD) ha propuestos un esquema para identificar el control glucémico de los pacientes diabéticos basado en las principales pruebas de laboratorio incluidas en su diagnóstico y seguimiento, glicemia en ayuna, glicemia pospandrial y hemoglobina glucosilada (ALAD, 2013).

Sin embargo, a pesar de contar con adelantos científico técnicos para diagnosticar precozmente la enfermedad y sus complicaciones, la tarea de mantener un control glucémico normal o adecuado en pacientes con DM está muy lejos de declararse como cumplida (Ramos Rangel, et al, 2017). Múltiples son los factores que pueden influir positiva o negativamente para alcanzar este objetivo; acciones encaminadas a lograr la adecuación de los esquemas terapéuticos, unido a una adecuada nutrición, sistematización de actividades físicas, aumento del nivel de conocimiento sobre autocuidado y la adherencia farmacológica deben ser incluidos en el seguimiento de los pacientes diabéticos (Couselo Fernández, \& Rumbo Prieto, 2018).
Se considera que muchos de estos factores no solo resultan vitales en el control de la enfermedad, sino que también constituyen elementos básicos para el debut de la enfermedad; de ahí que si se desea disminuir la prevalencia y la morbimortalidad de la DM se deben trazar estrategias encaminadas a generar hábitos y estilo de vida saludables como alternativa a la salud humana (Valladares Gonzalez, 2016; González Rodríguez, 2016).

Es por eso que teniendo en cuenta la creciente prevalencia de la DM, su influencia sobre la morbimortalidad nacional y en el contexto de investigación y la importancia de mantener un control glucémico normal o adecuado para disminuir la presencia de complicaciones; se decide realizar esta investigación con el objetivo de implementar una intervención terapéutica para elevar el control glucémico de los pacientes con DM atendidos en el HPGDR con deficiente control glucémico.

\section{METODOLOGÍA}

Se realizó una investigación aplicada, con un diseño cuasi experimental, alcance descriptivo y enfoque mixto. El universo estuvo constituido por 81 con historia previa de 2 controles glucémicos admisibles o inadecuados, según criterios de la ALAD, durante el año 2018. La investigación se llevó a cabo durante los meses de enero y julio del 2019.

Se aplicó la fórmula de cálculo muestral para poblaciones conocidas, la cual definió la muestra en un total de 73 pacientes y la composición se realizó mediante muestreo aleatorio simple. Los pacientes incorporados en el estudio debieron cumplir los criterios de 
inclusión definidos para la investigación.

Criterios de inclusión

- Pacientes adultos que asisten regularmente a consulta de seguimiento de su enfermedad de base.

- Expresar, mediante la firma del consentimiento informado, su deseo de participar en la investigación.

Para la investigación se definió como variable dependiente el control glucémico y como variables independientes el estado nutricional, nivel de conocimiento sobre autocuidado, adherencia farmacológica, práctica de actividades físicas y adecuación del esquema farmacológico. Como variables intervinientes se tuvieron en cuenta las características generales de los pacientes y las características clínicas de la enfermedad. La entrevista y la revisión documental fueron las técnicas de investigación utilizadas.

Se utilizó la escala de laALAD para determinar el control glucémico (ALAD, 2016), el índice de masa corporal (IMC) para determinar el estado nutricional de los pacientes (Guzmán Saldaña, et al, 2017), el cuestionario DKQ24 para identificar el nivel de conocimiento sobre autocuidado (Guerrero Pacheco, Galán Cuevas, \& Cappello, 2017; Zamora Niño, et al, 2019) y la guía terapéutica del Ministerio de Salud del Ecuador para analizar el estado del esquema farmacológico (MSP, 2016). Además se utilizó un modelo de recolección de datos para recopilar la información relacionada con las características generales de los pacientes y características clínicas de la DM.
La investigación se realizó en tres fases, una primera fase denominada pretest, donde además de solicitar el permiso de las autoridades se procedió a identificar los pacientes, componer la muestra y aplicar los cuestionarios e instrumentos. La segunda fase (intervención) consistió en la aplicación de la intervención que incluyó una seria de charlas que incluyeron los siguientes temas: importancia de la nutrición, la práctica sistemática de actividades físicas, adherencia terapéutica y autocuidado para lograr el control metabólico y disminuir las complicaciones. Cada charla educativa consto de 60 minutos de duración y fueron impartidas los días sábados; en cada actividad educativa se realizó una prueba exploratoria al inicio y una pregunta de comprobación de conocimientos al final de la actividad.

Para la realización de estas actividades se creó un equipo multidisciplinario constituido por especialista en medicina interna, nutricionista clínico, personal de enfermería y trabajadora social los cuales realizaron una valoración integral de cada paciente. Los temas de las charlas se decidieron a partir de los resultados del pretest. Para el control glucémico se utilizaron las cifras de hemoglobina glucosilada de los pacientes en cada uno de sus controles de seguimiento establecidos y recogidos en la historia clínica; la intervención no incluyó la realización de exámenes complementarios.

Las adecuaciones a los esquemas farmacológicos se realizaron en forma de sugerencias a los especialistas para que estos, conjuntamente con el paciente definieran la conducta definitiva en este sentido; 
los principales adecuaciones propuestas incluyeron la sustitución de la glibenclamida como fármaco hipoglucemiente oral en pacientes mayores de 70 años (riesgo de hipoglucemia) y la combinación de hipoglucemiantes orales (metformina) con dosis de insulina rápida en los pacientes que no alcanzaban el control glucémico adecuado con hipoglucemiantes orales solamente.

La tercera fase (postest), incluyó la aplicación nuevamente de los instrumentos utilizados en la investigación. A partir de los resultados alcanzados en el pretest y postest se pudo identificar la influencia ejercida por la intervención aplicada en el control glucémico de los pacientes que participaron en el estudio.

El procesamiento estadístico automatizado de la información se realizó mediante el programa estadístico SPSS (versión 20,5 para Windows). Se determinaron medidas de tendencia central y de dispersión para variables cuantitativas y frecuencias absolutas y porcentajes para variables cualitativas. Se utilizó la prueba no paramétrica de Rangos con Signos de Wilcoxon para identificar cambios en el control glucémico de los pacientes. Se definió el nivel de confianza en el 95\% y la significación estadística en un $\mathrm{p}$ igual o inferior a 0,05 . Los resultados obtenidos se mostraron en forma de tablas estadísticas para facilitar su comprensión.

Se cumplieron las normas y procedimientos de la declaración de Helsinki II para estudios en seres humanos, que incluyeron la voluntariedad y conocimiento de los pacientes sobre los elementos del estudio, el trato confidencial de los datos personales y la eliminación de la información una vez terminado el estudio.

\section{RESULTADOS Y DISCUSIÓN}

Se encontró un promedio de edad de 56,23 años con predominio de pacientes femeninas $(67,12 \%)$, con nivel educacional predominante de básico (38,35\%), casados $(78,08 \%)$ y con ingresos económicos catalogados como bajos (56,14\%). E1 80,82\% de los casos tenía diagnóstico de DM tipo 2, con un tiempo de evolución de la enfermedad predominante entre 5 y 10 años $(65,75 \%)$ y el $83,56 \%$ refirieron al menos una complicación de la enfermedad.

Tabla 1.- DISTRIBUCIÓN DE VARIABLES IDENTIFICADAS DURANTE EL PRETEST Y POSTEST.

\begin{tabular}{|c|c|c|c|c|}
\hline \multirow{3}{*}{$\begin{array}{l}\text { Variables incluidas en la } \\
\text { investigación }\end{array}$} & \multicolumn{4}{|c|}{ Resultados } \\
\hline & \multicolumn{2}{|c|}{ Pretest } & \multicolumn{2}{|c|}{ Pretest } \\
\hline & Frecuencia & Porciento & Frecuencia & Porciento \\
\hline \multicolumn{5}{|l|}{ Estado nutricional } \\
\hline Bajo peso & 4 & 5,48 & 3 & 4,11 \\
\hline Normo peso & 21 & 28,77 & 37 & 50,68 \\
\hline Sobre peso & 32 & 43,84 & 23 & 31,51 \\
\hline Obesidad & 16 & 21,92 & 10 & 13,70 \\
\hline \multicolumn{5}{|l|}{ Nivel de conocimiento } \\
\hline Bajo & 39 & 53,42 & 13 & 17,81 \\
\hline Medio & 26 & 35,62 & 41 & 56,16 \\
\hline Alto & 8 & 10,96 & 19 & 26,03 \\
\hline \multicolumn{5}{|l|}{ Adherencia farmacológica } \\
\hline Adherentes & 42 & 57,53 & 59 & 80,82 \\
\hline No adherentes & 32 & 42,47 & 14 & 19,18 \\
\hline \multicolumn{5}{|l|}{ Esquema farmacológico } \\
\hline Adecuado & 61 & 83,56 & 68 & 93,15 \\
\hline Inadecuado & 12 & 16,44 & 5 & 6,85 \\
\hline
\end{tabular}

Fuente: los autores de la investigación.

Se muestra en la tabla 1. que existió disminución considerable de los trastornos nutricionales por exceso al comparar los resultados del pretest y el postest. Se observa un aumento, aún insuficiente, en relación al nivel de conocimiento medio $(56,16 \%)$ y alto $(26,03)$ sobre autocuidado de la DM y en torno a la adherencia farmacológica que asciende hasta el $80,82 \%$ 
Tabla 2. RESULTADO DE LA PRUEBA DE RANGOS CON SIGNOS DE WILCOXON PARA EL CONTROL GLUCÉMICO COMPARANDO RESULTADOS DEL PRETEST Y POSTEST.

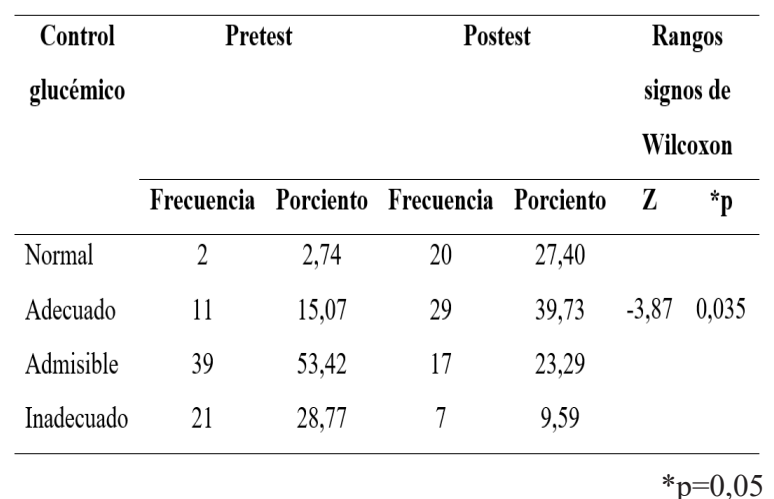

Fuente: los autores de la investigación.

En relación a la tabla 2. se observa un aumento estadísticamente significativo de pacientes con niveles de control glucémico normal y adecuado y disminución de casos en nivel admisible e inadecuado. Este resultado permite afirmar que existen evidencias para plantear que la intervención aplicada provocó cambios significativos en el mejoramiento del control glucémico de los pacientes diabéticos investigados.

\section{Discusión}

Las características generales de los pacientes coinciden con lo reportado internacionalmente donde se describe un predominio de pacientes femeninas (Sapunar 2016; Ramírez Hinojosa, et al, 2017). Las otras características generales estudiadas coinciden con la realidad ecuatoriana y de otros países en vías de desarrollo donde priman los niveles bajos de educación y de ingresos económicos (Puerta Rodríguez, 2010).

En relación a las características de la DM, se encontró predominio de pacientes con DM tipo 2, con tiempo de evolución de la enfermedad entre 5 y 10 años de edad y con presencia de complicaciones. Estos resultados han sido identificados por otros autores quienes incluso han reportado distintos grados de correlación entre ellas (Soler Sánchez, et al, 2016; Quiñones, et al, 2018; Solis Cartas, et al, 2020).

La aplicación de intervenciones de distintos tipos ha sido reportada como acciones favorables para lograr mejoras en distintos aspectos relacionados con el control glucémico de los pacientes diabéticos (Maidana, et al, 2017; Castillo Morejón, Martín Alonso, \& Almenares Rodríguez, 2017). En este sentido existen investigaciones que reportan resultados favorables en el mejoramiento de la adherencia farmacológica y control de la enfermedad (Mejia, et al, 2016; Zapata Zapata, Bergonzoli Peláez, \& Rodríguez, 2017; Cuenca Villalobos, et al, 2020).

Se describe que aumentar el nivel de conocimiento de los pacientes diabéticos constituye una herramienta de inestimable valor para los profesionales de la salud en su afán de controlar la glucemia en este tipo de pacientes. Al aumentar el nivel de conocimiento no solo se instruye al paciente a una alimentación saludable, sino que facilita la comprensión relacionada con la importancia de mantener una dieta balanceada, aporte adecuado de nutrientes y otros elementos relacionados con la nutrición (Jiménez León, et al, 2016; Abellán Ruiz, et al, 2017; Perulero Niño, Orozco González, \& Zúñiga Torres, 2019).

Otras investigaciones han adoptados distintos métodos de intervención sobre pacientes diabéticos relacionado con la práctica de actividades físicas (Soler Sánchez, 2016; 
Hernández, Arnold, \& Mendoza, 2018; Poblete Aro, et al, 2018). Se describe que mantener una actividad física adecuada no solo mejora la condición física, sino que también mejora el aspecto cardiovascular y respiratorio, disminuyendo el grado de afectación sistémica de la enfermedad (Souchay Díaz, et al, 2019)

Por último, es importante señalar como la intervención terapéutica aplicada abarcó una serie de elementos generales de los esquemas terapéuticos de la DM, este hecho refuerza la importancia de abordar integralmente la enfermedad como única alternativa al control clínico de la actividad de la misma y a la disminución de las complicaciones y la morbimortalidad por DM.

\section{CONCLUSIONES}

La intervención terapéutica implementada provocó cambios estadísticamente significativos en el mejoramiento del control glucémico de los pacientes objetos de la investigación; adicionalmente mejoró el nivel de conocimiento, el estado nutricional y la adherencia farmacológica de los pacientes.

\section{REFERENCIAS BIBLIOGRÁFICAS}

Abellán Ruiz, M.S., Barnuevo Espinosa, M.D., García Santamaría, C., Contreras Fernández, C.J., Aldeguer García, M., Soto Méndez, F., et al. Efecto del consumo de quinua (Chenopodium quinoa) como coadyuvante en la intervención nutricional en sujetos prediabéticos. Nutr. Hosp.,34(5),1163-1169. Recuperado de: $\quad$ http://scielo.isciii.es/scielo. php? script $=$ sci arttext\&pid $=\mathrm{S} 0212$ $16112017000500021 \& \operatorname{lng}=\mathrm{es}$

Alpízar, E., Zerquera Trujillo, G., Hernández Gutiérrez, C., \& Vicente Sánchez, B. (2017). Manejo práctico del paciente con diabetes mellitus en laAtención Primaria de Salud. Revista Finlay,7(1),104-125. Recuperado de: http://revfinlay.sld. cu/index.php/finlay/article/view/508

Asociación Latinoamericana de Diabetes (ALAD). Guía ALAD sobre el Diagnóstico, Control y Tratamiento de la Diabetes Mellitus Tipo 2 con Medicina Basada en Evidencia. Edición 2013,118-39.

Bell Castillo, J., George Carrión, W., García Céspedes, M.E., Delgado Bell, E., \& George Bell, M.J. (2017). Identification of metabolic syndrome in patients with diabetes mellitus and hypertension. MEDISAN, 1(10),3038-3045. Recuperado de http://scielo.sld.cu/scielo. php? script $=$ sci_arttext\&pid $=\mathrm{S} 1029$ $30192017001000007 \& 1 \mathrm{n}$ $\mathrm{g}=\mathrm{e} \mathrm{s} \& \mathrm{t} \quad \mathrm{n} \mathrm{g}=\mathrm{e} \mathrm{n}$ Castañeda Bajaña, E., García Martínez, M., Rebolledo Malpica, D., Muñiz Granoble, G., Calderón Vallejo, C., \& Álava Rengifo, N. (2019). Percepciones sobre adherencia del autocuidado desde la enfermería para pacientes con diabetes mellitus. Rev. Cubana De Enfermería,34(4),23-34. Recuperado de: http://www.revenfermeria.sld. cu/index.php/enf/article/view/3094

Castillo Morejón, M., Martín Alonso, 
L., \& Almenares Rodríguez, K. (2017). Adherencia terapéutica y factores influyentes en pacientes con diabetes mellitus tipo 2. Revista Cubana de Medicina General Integral,33(4),211-219. Recuperado de: $\quad$ http://scielo.sld.cu/scielo. php? script $=$ sci arttext\&pid $=$ S0864$21252017000400006 \& \operatorname{lng}=\mathrm{es} \& \operatorname{tlng}=\mathrm{es}$

Couselo Fernández, I., \& Rumbo Prieto, J.M. (2018). Riesgo de pie diabético y déficit de autocuidados en pacientes con Diabetes Mellitus Tipo 2. Enfermería universitaria,15(1),17-29. Disponible en: $\quad$ https://dx.doi.org/10.22201/ eneo.23958421e.2018.1.62902

Cuenca Villalobos, L., Uriarte Sandoval, M., Rodríguez Díaz, J., \& Parcon Bitanga, M. (2020). Uso de la medicina no convencional por pacientes diabéticos. Revista AMC,2020;24(1),245-253. Recuperado de: http://www.revistaamc.sld.cu/ index.php/amc/article/view/6632

González Rodríguez, R. (2016). La diabetes mellitus desde la Atención Primaria de Salud. MediCiego, 22(2):[aprox. 4 p.]. Disponible en: http://www.revmediciego.sld.cu/ index.php/mediciego/article/view/610

Guerrero Pacheco, R., Galán Cuevas, S., \& Cappello, O. (2017). Sociodemographic and psychological factors associated with self-care and quality of life in Mexican adults with type 2 Diabetes Mellitus. Acta Colombiana de Psicología,20(2),168-177.
Recuperado de: https://dx.doi. org/10.14718/ACP.2017.20.2.8

Guzmán Saldaña, R., Saucedo Molina, T.J., García Meraz, M., Galván García, M., \& Castillo Arreola, A. (2017). Imagen corporal e índice de masa corporal en mujeres indígenas del Estado de Hidalgo, México. Rev. Mex. de trastor. aliment.,8(1),56-62. Recuperado de: http://www.scielo.org.mx/scielo. php? script $=$ sci_arttext\&pid $=$ S2007$15232017000100056 \& \operatorname{lng}=\mathrm{es}$

Hernández, R.J., Arnold, D.Y., \& Mendoza, C.J. (2018). Efectos benéficos del ejercicio físico en las personas con diabetes mellitus tipo 2. Rev Cuba Endoc.,29(2),1-18. Recuperado de: https://www. medigraphic.com/cgi-bin/new/ resumen.cgi?IDARTICULO $=84725 \#$

Hospital Provincial General Docente de Riobamba (HPGDR). Sistema estadístico de causas de ingresos hospitalario. Dirección Zonal de Salud 3, Riobamba, Ecuador. 2018. Instituto Nacional de Estadística y Censo (INEC). Enfermedades Crónicas No Transmisibles en Ecuador. Ministerio de Salud Pública. Quito, Ecuador. 2018.

Instituto Nacional de Estadística y Censo (INEC). Registro nacional de nacimientos y Defunciones. Ministerio de Salud Pública. Quito, Ecuador. 2016.

Jiménez León, A.S., Pérez Escobar, I., Matías Pérez, D., \& García Montalvo, I.A. (2016). Intervención nutricional en un 
Grupo de Ayuda Mutua del municipio de “El Rosario”, Oaxaca de Juárez, México. Nutr. Hosp.,33(2),310-313. Recuperado de: $\quad$ http://scielo.isciii.es/scielo. php? script $=$ sci_arttext\&pid $=$ S0212$16112016000200020 \& \operatorname{lng}=\mathrm{es}$

Maidana, G.M., Zully, V., Samaniego, L., Acosta, P., Mastroianni, P., \& Lugo, G.B. (2017). Pharmaceutical interventions in patients with Type 2 Diabetes Mellitus. Ars Pharm,58(1),21-28. Recuperado de: $\quad$ http://scielo.isciii.es/scielo. php? script $=$ sci_arttext\&pid $=\mathrm{S} 2340$ $\underline{98942017000100021 \& \operatorname{lng}=\mathrm{es}}$

Martín Alfonso L. (2018). Adherencia terapéutica y factores influyentes en pacientes con diabetes mellitus tipo 2. Revista Cubana De Medicina General Integral,33(4):112-9. Recuperado de: http://revmgi.sld. cu/index.php/mgi/article/view/369

Mejía, M., Gil, Y., Quintero, O., \& D’Avila, M. (2016). Intervención educativa en el apego al tratamiento en pacientes con diabetes mellitus tipo 2. Trujillo. Venezuela. Revista Venezolana de Endocrinología y Metabolismo,16(1):34-46.

Ministerio de Salud Pública del Ecuador (MSP). Guía sobre el Diagnóstico, Control y Tratamiento de la Diabetes Mellitus. Quito, Ecuador, Ed. 2016.

Puerta Rodríguez, H. (2010). La crisis económica internacional y la integración. Opciones para los países subdesarrollados. Economía y Desarrollo, 145(1-2),48-71.
Recuperado de: https://www.redalyc. org/articulo.oa? id=425541314002

Perulero Niño, G., Orozco González, C.N., \& Zúñiga Torres, M.G. (2019). Intervenciones nutricionales para el tratamiento de la diabetes mellitus gestacional. Rev. Cienc. Salud,17(1),108-119. Recuperado de: $\quad$ http://www.scielo.org.co/scielo. php? script $=$ sci arttext\&pid $=\mathrm{S} 1692-$ $72732019000100108 \& \operatorname{lng}=\mathrm{en}$

Poblete Aro, C., Russell Guzmán, J., Parra, P., Soto Muñoz, M., Villegas González, B., Cofré Bola-Dos, C., et al. (2018). Efecto del ejercicio físico sobre marcadores de estrés oxidativo en pacientes con diabetes mellitus tipo 2. Rev. méd. Chile,146(3),362-372. Recuperado de: $\quad$ https://scielo.conicyt.cl/scielo. php? script $=$ sci arttext\&pid $=\mathrm{S} 0034-$ $98872018000300362 \& 1 \mathrm{ng}=\mathrm{es}$

Quiñones, Á., Ugarte, C., Chávez, C., \& Mañalich, J. (2018). Variables psicológicas asociadas a adherencia, cronicidadycomplicacionesenpacientes con diabetes mellitus tipo 2. Rev. méd. Chile,146(10),1151-1158. Recuperado de: $\quad$ https://scielo.conicyt.cl/scielo. php? script $=$ sci arttext\&pid $=$ S0034$\underline{98872018001001151 \& \operatorname{lng}=\mathrm{es}}$

Ramírez Hinojosa, J.P., Zacarías Castillo, R., Torres Tamayo, M., Tenorio Aguirre, E.K., \& Torres Viloria, A. Costos económicos en el tratamiento farmacológico del paciente con diabetes mellitus tipo 2. Estudio de pacientes en consulta externa de medicina interna 
de un hospital de segundo nivel de la Ciudad de México. Salud Pública de México,59(1),6-17. Recuperado de: https://dx.doi.org/10.21149/7944

Ramos Rangel, Y., Morejón Suárez, R., Gómez Valdivia, M., Reina Suárez, M., Rangel Díaz, C., \& Cabrera Macías, Y. (2017). Adherencia terapéutica en pacientes con diabetes mellitus tipo 2 . Revista Finlay,7(2),89-98. Disponible en: $\quad$ http://scielo.sld.cu/scielo. php? script $=$ sci_arttext\&pid $=\mathrm{S} 2221$ $\underline{24342017000200004 \& \operatorname{lng}=\mathrm{es} \& \text { tlng }=\mathrm{es}}$

SapunarJ.(2016).Epidemiologíadeladiabetes mellitus en Chile. Revista Médica Clínica Las Condes,27(2),146-151. Recuperado de: https://www. sciencedirect.com/science/ article/pii/S0716864016300037

Soler Sánchez, Y.M., Pérez Rosaba, E., López Sánchez, M.C., \& Quezada Rodríguez, D. (2016). Conocimientos y autocuidado en pacientes con diabetes mellitus tipo 2. Revista AMC,20(3),244-252. Recuperado de: https://www.redalyc. org/articulo.oa? id=211146067004

Solis Cartas, U.,Andramuño Núñez, V., Ávalos Obregón, M., Haro Chávez, J., Calvopiña Bejarano, S., Yambay Alulema, Á., \& Valdivieso Maggi, J. (2020). Relación entre tiroiditis autoinmune y lupus eritematoso sistémico. Rev Cubana de Reumatol.,22(1),e98. Recuperado de: http://www. revreumatologia.sld.cu/index.php/ reumatologia/article/view/715

Souchay Díaz, L., Sotolongo León, D.,
Alvarez Gavilán, Y., \& Castillo Duque, M. (2019). Complicaciones cardiovasculares y sus factores determinantes en pacientes adultos portadores de Enfermedad Renal Crónica. Panorama. Cuba y Salud,14(2(38)),23-31. Recuperado de: http://www.revpanorama.sld.cu/ index.php/panorama/article/view/862

Valladares Gonzalez, A.M. (2016). Enfoque psicológico del estilo de vida de los adultos medios diabéticos tipo II. Rev Cubana de Medicina General Integral.,32(4). Recuperado de: http://revmgi.sld. cu/index.php/mgi/article/view/210

Zamora Niño, C.F., Guibert Patiño, A.L., De La Cruz Saldaña, T., Ticse Aguirre, R., \& Málaga G. (2019). Evaluación de conocimientos sobre su enfermedad en pacientes con diabetes tipo 2 de un hospital de Lima, Perú y su asociación con la adherencia al tratamiento. Acta méd. Peru,36(2),96-103. Recuperado de: $\quad$ http://www.scielo.org.pe/scielo. php? script $=$ sci_arttext\&pid $=\mathrm{S} 1728$ $59172019000200004 \& 1 n g=e s$

Zapata Zapata, M.A., Bergonzoli Peláez, G., \& Rodríguez, A.L. (2017). Eficacia educacional en control metabólico de diabéticos con diálisis peritoneal. Revista Facultad Nacional de Salud Pública,35(1),49-57. Recuperado de https://dx.doi. org/10.17533/udea.rfnsp.v35n1a06 